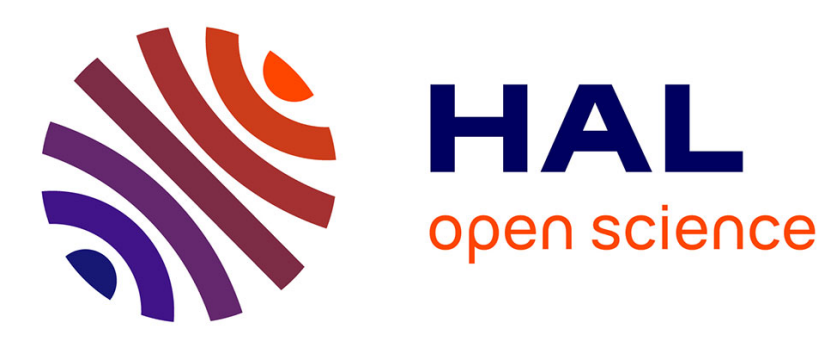

\title{
Multifractal analysis for cumulant-based epileptic seizure detection in eeg time series
}

Omar D Domingues, Philippe Ciuciu, Daria La Rocca, Patrice Abry, Herwig Wendt

\section{- To cite this version:}

Omar D Domingues, Philippe Ciuciu, Daria La Rocca, Patrice Abry, Herwig Wendt. Multifractal analysis for cumulant-based epileptic seizure detection in eeg time series. ISBI 2019 - IEEE International Symposium on Biomedical Imaging, Apr 2019, Venise, Italy. hal-02108099

\section{HAL Id: hal-02108099 \\ https://hal.inria.fr/hal-02108099}

Submitted on 24 Apr 2019

HAL is a multi-disciplinary open access archive for the deposit and dissemination of scientific research documents, whether they are published or not. The documents may come from teaching and research institutions in France or abroad, or from public or private research centers.
L'archive ouverte pluridisciplinaire HAL, est destinée au dépôt et à la diffusion de documents scientifiques de niveau recherche, publiés ou non, émanant des établissements d'enseignement et de recherche français ou étrangers, des laboratoires publics ou privés. 


\title{
MULTIFRACTAL ANALYSIS FOR CUMULANT-BASED EPILEPTIC SEIZURE DETECTION IN EEG TIME SERIES
}

\author{
Omar D. Domingues ${ }^{(1)}$, Philippe Ciuciu ${ }^{(1)}$, Daria La Rocca ${ }^{(1)}$, Patrice Abry $^{(2)}$ and Herwig Wendt ${ }^{(3)}$ \\ (1) CEA/NeuroSpin and INRIA-CEA Parietal, Univ. Paris-Saclay, France, philippe.ciuciuecea.fr \\ (2) Univ Lyon, Ens de Lyon, CNRS, Laboratoire de Physique, Lyon, France, patrice.abryeens-lyon.fr \\ (3) IRIT, CNRS, Univ. Toulouse, France, herwig.wendteirit.fr
}

\begin{abstract}
Multifractal analysis allows us to study scale invariance and fluctuations of the pointwise regularity of time series. A theoretically well grounded multifractal formalism, based on wavelet leaders, was applied to electroencephalography (EEG) time series measured in healthy volunteers and epilepsy patients, provided by the University of Bonn. We show that the multifractal spectrum during a seizure indicates a lower global regularity when compared to non-seizure data and that multifractal features, combined with few baseline features, can be used to train a supervised learning algorithm to discriminate well above chance ictal (i.e. seizure) versus healthy and interictal epochs $(\simeq 97 \%)$ and healthy controls versus patients $(\simeq 92 \%)$.
\end{abstract}

Index Terms - EEG, multifractal-analysis, seizure detection.

\section{INTRODUCTION}

Multifractal analysis is a useful tool to distinguish different behaviors of dynamic systems and stochastic processes. For instance, it has been applied to financial data for the comparison of price fluctuations of stocks and commodities [1], to texture classification [2] and the analysis of fetal heart rate variability [3], brain dynamics in functional neuroimaging [4, 5] among several other applications in physical and biological science. Multifractal analysis is intimately related to the notion of scale invariance of time series, which is often characterized by a Fourier power spectrum that behaves as a power law $|\nu|^{-\beta}$ in a range of frequencies $\nu \in\left[\nu_{1}, \nu_{2}\right]$, for $\beta>0$, which is referred to as power spectrum scale invariance. In multifractal analysis, scale invariance is defined in a more comprehensive way: whereas the Fourier power spectrum depends only on second-order statistics of the time series, multifractal analysis studies the power law behavior of $q$-th order statistics of multiresolution quantities denoted by $S(j, q)$ for each scale $j$ and for all $q \in \mathbb{R}$. Scale invariance is rede-

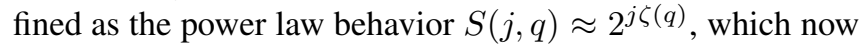

Work supported by ANR-16-CE33-0020 MultiFracs, France is described by a function $q \mapsto \zeta(q)$ instead of a single parameter $\beta$, providing a richer framework to scaling analysis. Moreover, the multifractal spectrum, which is theoretically estimated by a Legendre transform of $\zeta(q)$, can be shown to give us information regarding the overall pointwise regularity of a time series.

In this work, we used multifractal analysis - -based on the Wavelet Leader Multifractal Formalism (WLMF) [6] - to extract features from single channel EEG time series measured in healthy subjects and epileptic patients during seizure and seizure-free periods, using publicly available data provided by the University of Bonn [7]. We show that the average multifractal spectra are different between ictal (seizure) and interictal (between seizures) data, and also that multifractal features can be used to train a supervised learning algorithm to distinguish these two states with high accuracy. Also, these features were shown to be useful to discriminate healthy subjects and epileptic patients based on EEG data. Our approach does not focus on obtaining perfect classification scores: we aim to assess the usefulness of multifractal features in epileptic seizure detection, which may help understand neural dynamics during seizures.

In [8], the authors showed that multifractal features (also based on the WLMF) improved seizure prediction in mesial temporal lobe epilepsy using multi-channel intracanial EEG (iEEG) measurements, by training a classifier for each patient to discriminate preictal (state immediately before the seizure) and interictal epochs. The main advantages of the WLMF are: (i) it is theoretically well grounded and proved to be associated with the Hölder pointwise regularity and (ii) it is robust to smooth trends, since it is a wavelet-based method. Our contribution is the use of the WLMF to extract features from EEG and iEEG data measured from different subjects and different conditions to show that - even with this subject and measurement variability - multifractal features can discriminate well ictal versus healthy and interictal epochs and healthy subjects versus patients. 


\section{WAVELET-LEADER MULTIFRACTAL ANALYSIS}

The goal of multifractal analysis is to summarize the pointwise regularity of a time series $X(t)$ by estimating the Hausdorff dimension of the sets $\left\{t \in \mathbb{R}: h_{X}(t)=h\right\}$, denoted by $\mathcal{D}(h)$, where $h_{X}(t)$ is the Hölder exponent of $X(t)$ at time $t$. The function $h \mapsto \mathcal{D}(h)$ is called multifractal spectrum. In practice we only have access to $X(t)$ in a discrete set of time points, thus we cannot estimate $h_{X}(t)$ precisely, since it is a local quantity requiring the knowledge of $X(t)$ in arbitrarily small intervals centered at $t$. Multifractal formalisms allow us to estimate $\mathcal{D}(h)$ from multiresolution quantities which are easily computed for discretized signals. A theoretically well grounded formalism is based on wavelet leaders which are defined as follows: let $\psi_{0}(t)$ be a mother wavelet, let $\psi_{j, k}(t)=2^{-j} \psi_{0}\left(2^{-j} t-k\right)$, for $j, k \in \mathbb{Z}$ be dilated, translated and scaled versions of the mother wavelet and let $d_{X}(j, k)=\int_{\mathbb{R}} X(t) \psi_{j, k}(t) \mathrm{d} t$ be the wavelet coefficients of $X(t)$. The wavelet leaders of $X(t)$ at scale $j$ and time $k$ are defined as the supremum of the wavelet coefficients at scales inferior to $j$ and in a time window around $k$ :

$$
L_{X}(j, k)=\sup _{j^{\prime} \leq j, 2^{j^{\prime}} k^{\prime} \in 3 \lambda_{j, k}}\left|2^{j^{\prime} \gamma} d_{X}\left(j^{\prime}, k^{\prime}\right)\right|
$$

where $\lambda_{j, k}=\left[k 2^{j},(k+1) 2^{j}\right), 3 \lambda_{j, k}=\lambda_{j, k-1} \cup \lambda_{j, k} \cup$ $\lambda_{j, k+1}$ and $\gamma>0$ is a parameter that ensures that minimum regularity assumptions on the data are met, cf. [2] for details. Multifractal analysis is related to the scale invariance of the structure functions $S(j, q)$, which are time averages of the $q$ th power of wavelet leaders at each scale. Scale invariance is defined as follows:

$$
S(j, q):=\frac{1}{n_{j}} \sum_{k=1}^{n_{j}}\left|L_{X}(j, k)\right|^{q} \approx 2^{j \zeta(q)}
$$

for $j \in\left[j_{1}, j_{2}\right], q \in \mathbb{R}$, where $n_{j}$ is the number of wavelet leaders available at scale $j$ and $\zeta(q)$ is referred to as scaling function. It can be proven that the Legendre transform of $\zeta(q)$ is an upper bound of the multifractal spectrum $\mathcal{D}(h)$ :

$$
\mathcal{L}(h):=\inf _{q \in \mathbb{R}}(1+q h-\zeta(q)) \geq \mathcal{D}(h) .
$$

The function $h \mapsto \mathcal{L}(h)$ is called Legendre spetrum and it is possible to estimate $\mathcal{L}(h)$ without computing a numerical Legendre transform. Consider the first two cumulants of $\log L_{X}(j, \cdot)$, denoted by $C_{1}(j)$ and $C_{2}(j)$, which are computed for all $j \in\left[j_{1}, j_{2}\right]$, the range of scales where we observe a scale-invariant behavior:

$$
\begin{gathered}
C_{1}(j)=\frac{1}{n_{j}} \sum_{k=1}^{n_{j}} \log _{2} L_{X}(j, k) \\
C_{2}(j)=\frac{1}{n_{j}} \sum_{k=1}^{n_{j}}\left(\log _{2} L_{X}(j, k)\right)^{2}-C_{1}(j)^{2} .
\end{gathered}
$$

It has been shown [2] that if the cumulants behave linearly as a function of the scale $j$, i.e. $C_{1}(j)=a+c_{1} j$ and $C_{2}(j)=b+c_{2} j$, we have the approximation $\mathcal{L}(h) \approx$ $1+\left(h-c_{1}\right)^{2} /\left(2 c_{2}\right)$ if $c_{2}<0$. Thus, if $c_{2}<0$ the time series presents a multifractal behavior. The coefficients $c_{1}$ and $c_{2}$ are called log-cumulants; $c_{1}$ represents the most frequent regularity parameter in the time series, whereas $c_{2}$ measures the amount of variability in this regularity.

\section{EEG DATA AND EXPERIMENTAL DESIGN}

An open source Python toolbox ${ }^{1}$ was used to apply multifractal analysis to the publicly available EEG dataset provided by a research group at the University of Bonn [7]. This dataset consists of 5 sets (A, B, C, D and E) with 100 time series each. Sets A and B contain scalp-based EEG measurements collected in five healthy volunteers. Volunteers were kept with eyes open (set A) or closed (set B). Sets C, D and E contain presurgical intracranial EEG (iEEG) measurements recorded in five patients; time series in sets $\mathrm{C}$ and $\mathrm{D}$ were recorded during a seizure-free (interictal) interval whereas time series in set $\mathrm{E}$ were recorded during an epileptic seizure (ictal). According to [7], data in set $\mathrm{D}$ were measured from within the epileptogenic zone, whereas data in set $\mathrm{C}$ were measured from the hippocampal formation of the opposite hemisphere of the brain. All time series are 4097 time-point long with a sampling frequency of $173.61 \mathrm{~Hz}$, corresponding to a recording duration of 23.6 seconds. Fig. 1 shows examples of one time series of each set.

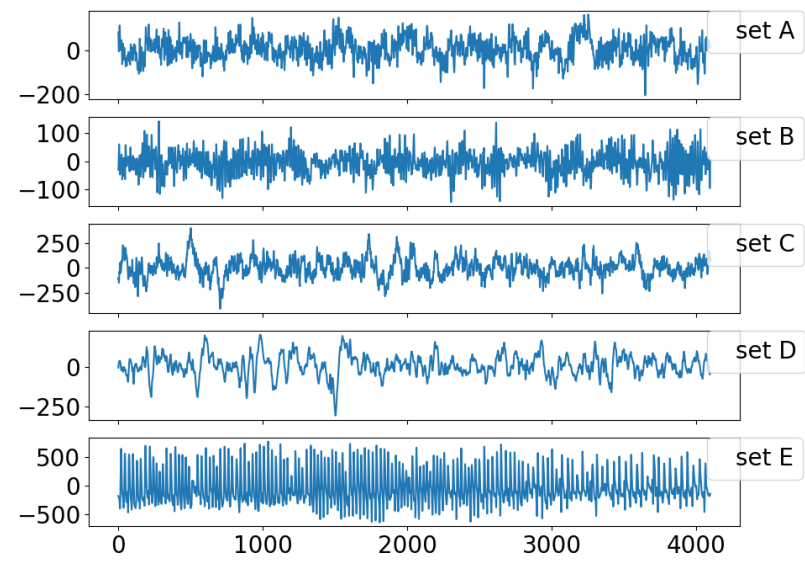

Fig. 1. Examples of time series in Bonn dataset. Sets A and B: EEG of healthy volunteer; sets C and D: iEEG for a patient during a seizure-free interval; set E: iEEG for a patient during epileptic seizure.

The wavelet leaders were computed over each of the 500 time series. We observed that the relation (2) holds in the range of scales $\left[j_{1}, j_{2}\right]=[5,9]$, which corresponds to time scales between $0.1 \mathrm{~s}$ and $3 \mathrm{~s}$ approximately. In this range of scales, we used the relations (4)-(5) to compute the $\log$-cumulants $c_{1}$

\footnotetext{
${ }^{1}$ https://github.com/neurospin/mfanalysis, see also [5]
} 
and $c_{2}$. The parameter $\gamma$ was set to 1.6 to ensure minimum regularity assumptions for all time series.

The cumulants $c_{1}$ and $c_{2}$ were then used as features to train a random forest classifier ${ }^{2}$ for three classification problems:

- ABCD vs. E: Discriminate seizure (set E) from nonseizure (sets A, B, C and D) time series;

- AB vs. CDE: Discriminate EEG of healthy subjects (sets A and B) from EEG of epilepsy patients (sets $\mathrm{C}, \mathrm{D}$ and $\mathrm{E}$ );

- AB vs. CD vs. E: Discriminate between healthy subjects, patients in seizure-free interval and patients during a seizure.

Other features were also used for comparison: peakto-peak amplitude, skewness, kurtosis and line length [10], which were computed directly from each time series using the mne-features library for Python [11]. Precisely, we compared seven sets of features:

- Feature set 1: $c_{1}$ only;

- Feature set 2: $c_{2}$ only;

- Feature set 3: $c_{1}$ and $c_{2}$;

- Feature set 4: peak-to-peak amplitude;

- Feature set 5: peak-to-peak amplitude, skewness, kurtosis and line length;

- Feature set 6: $c_{1}, c_{2}$ and peak-to-peak amplitude;

- Feature set 7: $c_{1}, c_{2}$, peak-to-peak amplitude, skewness, kurtosis and line length.

\section{RESULTS AND DISCUSSION}

Fig. 2 shows the average Legendre spectrum computed for all time series in each of the sets A-E. Interestingly, the average Legendre spectrum for set $\mathrm{E}$ is shifted to the left of all other average spectra, which reflects a lower global regularity in the data set $\mathrm{E}$ as compared to others. Fig. 3 shows the values of $c_{1}$ and $c_{2}$ for all time series, and we can see that the seizure (set E) and the non-seizure (sets A, B, C and D) data are approximately separated into two different clusters. Also, $c_{2}<0$ for $88 \%$ of seizure data and for $96 \%$ of seizure-free data, meaning that most time series presented a multifractal behavior.

For each classification problem and each set of features, we computed the accuracy and the area under the receiver operating characteristic curve (AUROC) for a 15-fold crossvalidation scheme, in which data were randomly split 15 times into a training set containing 300 time series and a test set containing 200 time series. We report the average accuracy and AUROC and their standard deviations. We used a random forest consisting of 100 classification trees and no hyperparameter was optimized, to avoid possible over-optimistic results.

\footnotetext{
${ }^{2}$ Using the scikit-learn library for Python [9].
}

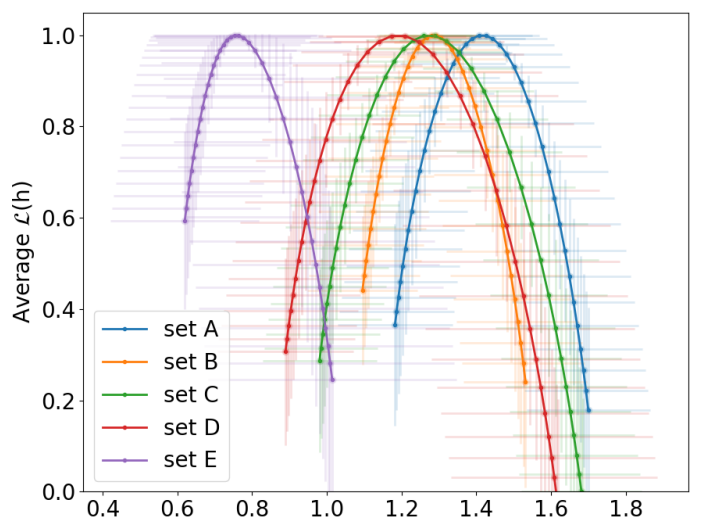

Fig. 2. Average Legendre spectrum $\mathcal{L}(h)$ for all time series in each data set. Error bars represent \pm one standard deviation.

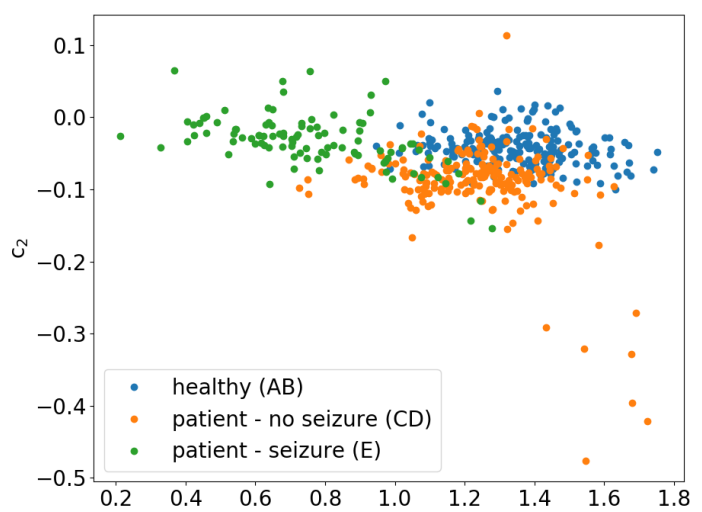

Fig. 3. Visualization of $c_{1}$ and $c_{2}{ }^{c_{1}}$ for all time series in all sets A, B, C, D and E.

Table 1 shows the accuracy and the AUROC obtained for the classification of $\mathrm{ABCD}$ vs. E. Considering that the chance level accuracy of this problem is $80 \%$, most feature sets perform well (accuracy $>91.5 \%$ ) when compared to chance. Using only $c_{1}$ and $c_{2}$ as features gives us a $94.37 \%$ accuracy, compared to $93.57 \%$ using only the peak-to-peak amplitude (feature set 4) and $96.87 \%$ using the peak-to-peak amplitude combined with the skewness, kurtosis and line length (feature set 5). When we add $c_{1}$ and $c_{2}$ to the features sets 4 and 5 , the accuracy in both cases is increased to $97.03(+3.46 \%)$ and to $97.40 \%(+0.53 \%)$, respectively. Although the accuracy and AUROC obtained with the feature set 5 (96.87\% and 0.995) is not considerably increased when we add $c_{1}$ and $c_{2}(97.40 \%$ and 0.997 ), we obtain almost the same results with feature set 6 (97.03\% and 0.994), which uses only $c_{1}, c_{2}$ and the peakto-peak amplitude, a very simple feature.

Table 2 shows the accuracy and AUROC obtained for the classification of $\mathrm{AB}$ (healthy subjects) vs. $\mathrm{CDE}$ (epilepsy patients). The chance level accuracy is this case is $60 \%$, and we obtain a $84.90 \%$ accuracy and a 0.919 AUROC using $c_{1}$ and $c_{2}$ (feature set 3 ). With feature set 5 (peak-to-peak, skewness, kurtosis, line length) we obtain $88.40 \%$ and 0.959 , which are increased to $92.57 \%(+4.17 \%)$ and $0.975(+0.016)$ if we include $c_{1}$ and $c_{2}$ (feature set 7 ). 
Table 1. ABCD vs. E - Accuracy and AUROC

\begin{tabular}{c|c|c}
\hline Feature Set & Accuracy (\%) & AUROC \\
\hline $\mathbf{1}$ & $91.53 \pm 1.75$ & $0.955 \pm 0.017$ \\
$\mathbf{2}$ & $79.37 \pm 1.90$ & $0.724 \pm 0.033$ \\
$\mathbf{3}$ & $94.37 \pm 0.90$ & $0.960 \pm 0.025$ \\
$\mathbf{4}$ & $93.57 \pm 1.39$ & $0.974 \pm 0.008$ \\
$\mathbf{5}$ & $96.87 \pm 0.81$ & $0.995 \pm 0.002$ \\
$\mathbf{6}$ & $97.03 \pm 0.97$ & $0.994 \pm 0.005$ \\
$\mathbf{7}$ & $\mathbf{9 7 . 4 0} \pm \mathbf{0 . 8 6}$ & $\mathbf{0 . 9 9 7} \pm \mathbf{0 . 0 0 2}$ \\
\hline
\end{tabular}

Table 2. AB vs. CDE - Accuracy and AUROC

\begin{tabular}{c|c|c}
\hline Feature Set & Accuracy (\%) & AUROC \\
\hline $\mathbf{1}$ & $69.77 \pm 1.91$ & $0.774 \pm 0.020$ \\
$\mathbf{2}$ & $66.17 \pm 2.30$ & $0.727 \pm 0.028$ \\
$\mathbf{3}$ & $84.90 \pm 1.75$ & $0.919 \pm 0.011$ \\
$\mathbf{4}$ & $66.30 \pm 1.43$ & $0.724 \pm 0.014$ \\
$\mathbf{5}$ & $88.40 \pm 3.27$ & $0.959 \pm 0.011$ \\
$\mathbf{6}$ & $86.13 \pm 1.90$ & $0.934 \pm 0.009$ \\
$\mathbf{7}$ & $\mathbf{9 2 . 5 7} \pm \mathbf{1 . 9 7}$ & $\mathbf{0 . 9 7 5} \pm \mathbf{0 . 0 0 9}$ \\
\hline
\end{tabular}

Table 3 shows the accuracy obtained for the classification of $\mathrm{AB}$ vs. $\mathrm{CD}$ vs. $\mathrm{E}^{3}$. The chance level accuracy in this case is $40 \%$. Using $c_{1}$ and $c_{2}$ only, we obtain a $80.93 \%$ accuracy and $86.03 \%$ with feature set 5 (peak-to-peak, skewness, kurtosis, line length). With feature set 7 , the accuracy is increased to $90.37 \%(+4.34 \%$ with respect to set 5$)$.

\begin{tabular}{|c|c|}
\hline Feature Set & Accuracy $(\%)$ \\
\hline 1 & $61.13 \pm 2.51$ \\
\hline 2 & $62.57 \pm 1.99$ \\
\hline 3 & $80.93 \pm 1.83$ \\
\hline 4 & $61.70 \pm 2.06$ \\
\hline 5 & $86.03 \pm 2.65$ \\
\hline 6 & $83.97 \pm 1.70$ \\
\hline 7 & $90.37 \pm 1.79$ \\
\hline
\end{tabular}

These results show that $c_{1}$ and $c_{2}$ perform well above chance when used to discriminate seizure versus non-seizure and healthy versus patient time series. Although feature set 5 (peak-to-peak, skewness, kurtosis, line length) performed better than the log-cumulants alone, $c_{1}$ and $c_{2}$ where shown to improve accuracy when combined to those features.

\section{CONCLUSION AND PERSPECTIVES}

Our results show that $c_{1}$ and $c_{2}$, which are features that characterize the multifractal spectrum of a time series, are relevant for the detection of epileptic seizures as well as for dis- criminating healthy volunteers from patients. These features can be seen as a way to summarize the dynamics of time series: $c_{1}$ represents the overall regularity of the signal while $c_{2}$ measures the variability around this global regularity. This suggests that the neural dynamics summarized by $c_{1}$ and $c_{2}$ can be modulated by seizure (ictal) activity and can be also different between healthy subjects and epileptic patients. Future work should investigate whether these conclusions can be extended to other data sets and whether we can propose computational neuroscience models that explain the modulation of multifractal properties, aiming to better understand the neural dynamics involved in epilepsy.

\section{REFERENCES}

[1] K. Matia, Y. Ashkenazy, and H. E. Stanley, "Multifractal properties of price fluctuations of stocks and commodities," EPL (Europhysics Letters), vol. 61, pp. 422-428, Feb. 2003.

[2] H. Wendt, S. Roux, P. Abry, and S. Jaffard, "Wavelet leaders and bootstrap for multifractal analysis of images," Signal Proces., vol. 89, no. 6, pp. 1100-1114, 2009.

[3] M. Doret, H. Helgason, P. Abry, P. Goncalves, C. Gharib, and P. Gaucherand, "Multifractal Analysis of Fetal Heart Rate Variability in Fetuses with and without Severe Acidosis during Labor," American Journal of Perinatology, vol. 28, no. 4, pp. 259-266, Apr 2011.

[4] P. Ciuciu, G. Varoquaux, P. Abry, S. Sadaghiani, and A. Kleinschmidt, "Scale-Free and Multifractal Time Dynamics of fMRI Signals during Rest and Task.," Frontiers in physiology, vol. 3, no. Article 186, pp. 1-18, June 2012.

[5] D. La Rocca, N. Zilber, P. Abry, V. van Wassenhove, and P. Ciuciu, "Self-similarity and multifractality in human brain activity: a waveletbased analysis of scale-free brain dynamics," Journal of Neuroscience Methods, vol. 309, pp. 175-187, Nov. 2018.

[6] H. Wendt, P. Abry, and S. Jaffard, "Bootstrap for empirical multifractal analysis," IEEE Signal Proc. Mag., vol. 24, no. 4, pp. 38-48, 2007.

[7] R. G. Andrzejak, K. Lehnertz, F. Mormann, C. Rieke, P. David, and C. E. Elger, "Indications of nonlinear deterministic and finitedimensional structures in time series of brain electrical activity: Dependence on recording region and brain state," Phys. Rev. E, vol. 64, pp. 061907, Nov 2001.

[8] K. Gadhoumi, J. Gotman, and J. M. Lina, "Scale invariance properties of intracerebral eeg improve seizure prediction in mesial temporal lobe epilepsy," PLOS ONE, vol. 10, no. 4, pp. 1-23, 042015.

[9] F. Pedregosa, G. Varoquaux, A. Gramfort, V. Michel, B. Thirion, O. Grisel, M. Blondel, P. Prettenhofer, R. Weiss, V. Dubourg, J. Vanderplas, A. Passos, D. Cournapeau, M. Brucher, M. Perrot, and E. Duchesnay, "Scikit-learn: Machine learning in Python," Journal of Machine Learning Research, vol. 12, pp. 2825-2830, 2011.

[10] R. Esteller, J. Echauz, T. Tcheng, B. Litt, and B. Pless, "Line length: an efficient feature for seizure onset detection," in 2001 Conference Proceedings of the 23rd Annual International Conference of the IEEE Engineering in Medicine and Biology Society, Oct 2001, vol. 2, pp. $1707-1710$ vol.2.

[11] J.-B. Schiratti, J.-E. Le Douget, M. Le Van Quyen, S. Essid, and A. Gramfort, "An ensemble learning approach to detect epileptic seizures from long intracranial EEG recordings," in International Conference on Acoustics, Speech, and Signal Processing, Calgary, Canada, Apr. 2018.

\footnotetext{
lem.

${ }^{3}$ The AUROC was not computed for this multiclass classification prob-
} 